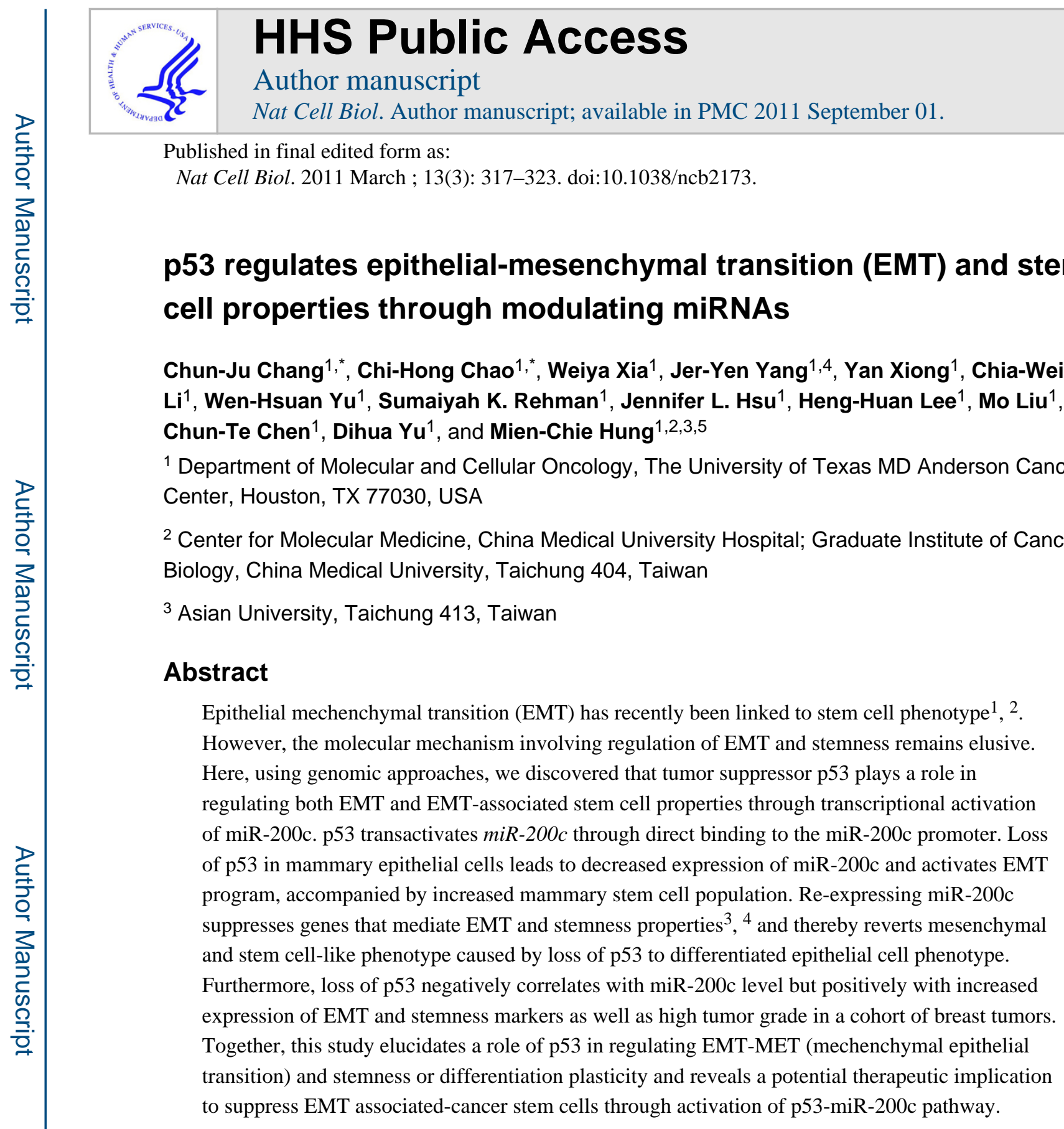

Published in final edited form as:

Nat Cell Biol. 2011 March ; 13(3): 317-323. doi:10.1038/ncb2173.

\title{
p53 regulates epithelial-mesenchymal transition (EMT) and stem cell properties through modulating miRNAs
}

\author{
Chun-Ju Chang , Chi-Hong Chao ${ }^{1,}$, Weiya Xia , Jer-Yen Yang ${ }^{1,4}$, Yan Xiong ${ }^{1}$, Chia-Wei \\ $\mathrm{Li}^{1}$, Wen-Hsuan $\mathrm{Yu}^{1}$, Sumaiyah K. Rehman ${ }^{1}$, Jennifer L. Hsu${ }^{1}$, Heng-Huan Lee ${ }^{1}$, Mo Liu ${ }^{1}$, \\ 1 Department of Molecular and Cellular Oncology, The University of Texas MD Anderson Cancer \\ Center, Houston, TX 77030, USA \\ ${ }^{2}$ Center for Molecular Medicine, China Medical University Hospital; Graduate Institute of Cancer \\ Biology, China Medical University, Taichung 404, Taiwan \\ Asian University, Taichung 413, Taiwan
}

\begin{abstract}
Epithelial mechenchymal transition (EMT) has recently been linked to stem cell phenotype ${ }^{1}{ }^{2}$. However, the molecular mechanism involving regulation of EMT and stemness remains elusive. Here, using genomic approaches, we discovered that tumor suppressor p53 plays a role in regulating both EMT and EMT-associated stem cell properties through transcriptional activation of miR-200c. p53 transactivates $m i R-200 c$ through direct binding to the miR-200c promoter. Loss of p53 in mammary epithelial cells leads to decreased expression of miR-200c and activates EMT program, accompanied by increased mammary stem cell population. Re-expressing miR-200c suppresses genes that mediate EMT and stemness properties ${ }^{3},{ }^{4}$ and thereby reverts mesenchymal and stem cell-like phenotype caused by loss of p53 to differentiated epithelial cell phenotype. Furthermore, loss of p53 negatively correlates with miR-200c level but positively with increased expression of EMT and stemness markers as well as high tumor grade in a cohort of breast tumors. Together, this study elucidates a role of p53 in regulating EMT-MET (mechenchymal epithelial to suppress EMT associated-cancer stem cells through activation of p53-miR-200c pathway.
\end{abstract}

\footnotetext{
Users may view, print, copy, download and text and data- mine the content in such documents, for the purposes of academic research, subject always to the full Conditions of use: http://www.nature.com/authors/editorial_policies/license.html\#terms

${ }^{5}$ Correspondence should be addressed to M.-C.H., Phone: (713) 792-3668. Fax: (713) 794-3270. mhung@mdanderson.org.

C.-J. Chang and C.-H. Chao contributed equally to this work.

${ }^{4}$ Present address: Department of Developmental Biology, Stanford University, Stanford, CA 94305

Author Contributions

C.-J.C., C.-H.C. and M.-C.H. designed and conceived the study. C.-J.C. and C.-H.C. performed the experiments and wrote the manuscript. W.X. performed and analyzed the immunohistochemistry experiments. Y.X., S.K.R., D.Y. performed the miRNA in situ hybridization experiments. J.-Y.Y., C-W.L., W.-H.Y., H.-H.L., M.L. helped with the biochemical experiments. C-T.C. and H.J.L. helped with the animal experiments. H.J.L. edited the manuscript.

We have no competing financial interests.

Accession Numbers

MicroRNA array data is deposited in NCBI GEO database (GSE 25037).
} 
The epithelial-mesenchymal transition (EMT) and the reverse process, termed the mesenchymal-epithelial transition (MET), are key programs in regulating embryogenesis ${ }^{5}$. There is evidence to suggest that aberrant EMT activation also contributes to cancer progression and metastasis ${ }^{2}$. Recent findings further illustrate a link between EMT and the gain of stem cell properties in normal and neoplastic cell populations ${ }^{1}{ }^{2}$.

Micro-RNAs (miRNAs), the small non-coding RNA molecules that suppress gene expression by interacting with the $3^{\prime}$ untranslated regions ( $3^{\prime}$ UTRs) of target mRNAs, have also been linked to EMT and cancer ${ }^{3}$. Notably, miR-200c was shown to regulate EMT through inhibiting ZEB1/2, transcription repressors of E-cadherin, a known epithelial cell marker. Reports have also indicated that miR-200c is downregulated in normal stem cell and neoplastic stem cell population as it suppresses BMI1, a Polycomb protein that is involved the maintenance of stemness properties ${ }^{3},{ }^{4}$. All these results suggest that miR-200c may play an integral role in modulating EMT and stem cell phenotype.

Despite the efforts in studying EMT and stem cell properties as mentioned above, regulation of the plasticity of EMT-stemness is not clear and molecular mechanism that regulates miR-200c or other stemness-related miRNAs is also largely unknown. Thus, we first attempted to investigate miRNA expression profiles in $\mathrm{CD} 24^{-} \mathrm{CD} 44^{+}$(stem) and non$\mathrm{CD} 24^{-} \mathrm{CD} 44^{+}$(non-stem) cell populations isolated from primary human mammalian epithelial cells (HMECs) and normal mammary epithelial cell line MCF12A, both of which have been used to study EMT or/and stem cell properties ${ }^{3},{ }^{11}$. By screening a genome wide microRNA-array, miRNAs which show significant differences in expression levels between stem and non-stem cell populations in both cells lines (Supplementary Information, Table S1a) were further validated using qPCR analysis (Fig. 1a). Among these miRNAs, miR-183 and miR-200c were the most down-regulated in the stem cell population compared to the non-stem cell population ( $>2$-fold change). Consistently, miR-183 and miR-200c were also identified as the most down-regulated miRNAs in the stem cell population using a validated PCR array consisting of $\sim 90$ annotated miRNAs that are related to cancer and metastasis (SA Biosciences, data not shown). To further investigate potential regulatory mechanism of these two miRNAs, we analyzed the response elements of a cohort of transcription factors located within $2 \mathrm{~kb}$ region upstream of the transcription starting site of miR-183 and miR-200c using promoter analysis. Among all the transcription factor-response elements, p53 response element was the one that overlaps in both miR-183 and miR-200c promoter regions with the highest consensus score of $\searrow 0.98$ for both miRNAs (matrix similarity score $>0.95$ as cutoff, score $=1$ as perfect match) (Fig. 1b, Supplementary Information, Table S2a and S2b). To validate direct association of p53 with miR-200c and miR-183 promoters, we performed chromatin immunoprecipitation (ChIP) analysis in HMEC cells for all the putative p53 consensus binding elements within miR-200c and miR-183 promoters (Fig. 1b, element A-E) using an antibody specifically against p53. The ChIP results revealed that p53 is most significantly bound to element $C$ within miR-200c promoter and element $E$ within miR-183 promoter (Fig. 1c). Knocking-down p53 diminished the amount of DNA (element $\mathrm{C}$ and $\mathrm{E}$ ) that could be immunoprecipitated by p53 antibody (Fig. 1d), suggesting p53 directly and specifically associates with these promoter regions. Furthermore, upregulation of $\mathrm{p} 53$ by etoposide resulted in transcription activation of the luciferase expression driven by miR-200c and miR 183 promoters, which could be impaired by mutations of the p53 
response elements ( $\mathrm{C}$ and $\mathrm{E}$ ) (Fig. 1e). Consistently, ectopic expression of $\mathrm{p} 53$ in both HMEC and MCF12A cells significantly increased expression of miR-200c and miR183 in both cell lines (Fig. 1f). miR-200b was used as a negative control as it was not regulated by p53 (Fig. 1f, Supplementary Information, Table S1b). Furthermore, knocking-down p53 markedly reduced levels of miR-200c and miR-183 (Fig. 1g, Supplementary Information, Table S1) and increased levels of KLF4 and BMI1, two stemness-associated genes that are known RNA targets of miR-200c and miR- $183^{4}$ (Fig. 1g). Together, these data suggest that p53 binds to specific promoter elements of miR-200c and miR-183, where p53 activates the transcription of miR-200c and miR-183.

Given the association of certain miRNAs, e.g. miR-183 and miR-200c, with EMT and stem cell properties in previous studies ${ }^{3},{ }^{4}$, we hypothesized that p53 may play a role in regulating EMT plasticity and stem cell properties through regulating these miRNAs. To test this hypothesis, we first examined p53 expression levels along with the EMT marker expression levels during EMT process. We treated MCF12A cells with TGF- $\beta$ for 7 days, a standard treatment that induces EMT ${ }^{3}$. Similar to previous observations, TGF- $\beta$ treatment induced EMT phenotype from a cobblestonestone-like to a spindle-like morphology (Supplementary Information, Fig. S1a), accompanied by decreased expression of E-cadherin (Supplementary Information, Fig. S1b). Interestingly, p53 expression level was also significantly reduced during TGF- $\beta$ induced EMT process (Supplementary Information, Fig. S1b). Next, we asked whether expression levels of the p53 regulated-miRNAs are also altered by TGF- $\beta$ treatment. We found that like p53, miR-183 and miR-200c are downregulated whereas two of its RNA targets- BMI1 and KLF4 are upregulated in TGF $\beta$ induced EMT cells (Supplementary Information, Fig. S1c). Since miR-200c shows much more significant response upon TGF- $\beta$ treatment compared to miR-183 (Supplementary Information, Fig. S1c), we will later focus on miR-200c as the prime target of p53 regulation. Together, these data suggest that p53 expression physiologically associates with miRNAs that are involved in the regulation of EMT and stem cell properties.

To understand whether p53 can directly regulate EMT process and EMT-associated stem cell properties, we first knocked-down p53 in MCF12A cells using three individual shRNAs (Fig. 2 and Supplementary Information, Fig. S1d-f). Similar to TGF- $\beta$ treatment (Supplementary Information, Fig. S1a-c) reduction of p53 expression converted predominant epithelial phenotype to evident EMT phenotype (EMT) (Fig. 2a), accompanied by a decrease of epithelial cell marker, such as E-cadherin and an increase of mesenchymal cell markers, such as N-cadherin, Vimentin, and ZEB1 (Fig. 2b, Supplementary Information, Fig. S1d and S1e). Next, we asked whether increased expression of p53 could revert the mechenchymal phenotype to epithelial phenotype (MET), we expressed p53-GFP in TGF- $\beta$ treated-MCF12A cells and examined E-cadherin expression in the isolated GFP+ cell populations. In Supplementary Information Fig. S1a-b, both p53 and E-cadherin were downregulated upon TGF- $\beta$ treatment. Here, we found that enhanced $\mathrm{p} 53$ expression by ectopic expression (Supplementary Information, Fig. S2a) or by etoposide treatment (Supplementary Information, Fig. S2c) is able to revert TGF- $\beta$ induced mesenchymal phenotype to epithelial phenotype (Supplementary Information, Fig. S2b) and block Ecadherin suppression mediated by TGF- $\beta$ treatment (Supplementary Information, Fig. S2a and S2d). Consistently, in an established HMEC-Snail cell line ${ }^{3}$, which shows predominant 
mensenchymal phenotype, re-expression of p53 led to significant elevation of E-cadherin expression (Fig. 2c). These data suggest that p53 may regulate EMT-MET plasticity.

To elucidate whether p53 could also influence EMT-associated stem cell properties, we knocked-down p53 in MCF12A cells. Consistent with the previous results showing loss of epithelial phenotype by knocking-down p53 (Fig. 2a), reduced p53 expression resulted in significant elevation of the $\mathrm{CD} 24^{-} \mathrm{CD} 44^{+}$stem cell population (Fig. 2d, Supplementary Information, Fig. S1f) and enhanced BMI1 expression (Fig. 2b, Supplementary Information, Fig. S1e). To determine whether increased expression of p53 could repress stem cell population enhanced by TGF- $\beta$ treatment, we expressed p53-GFP in TGF- $\beta$ treatedMCF12A cells and examined abundance of the $\mathrm{CD} 24^{-} \mathrm{CD} 44^{+}$stem cell population in the isolated GFP+ cell populations (Supplementary Information, Fig. S3a). Indeed, p53 expression blocked the increase of stem cell population induced by TGF- $\beta$ treatment (Supplementary Information, Fig. S3a). Furthermore, in HMEC-Snail cells, which showed high abundance of $\mathrm{CD} 24^{-} \mathrm{CD} 44^{+}$stem cell population, re-expression of p53 also significantly decreased $\mathrm{CD} 24^{-} \mathrm{CD} 44^{+}$cells (Fig. 2e). These data suggest that p53 may suppress EMT-associated stem cell properties.

To further understand whether p53 regulates EMT-MET transition through modulating miR-200c, we expressed precursor miR-200c in p53 knocked-down MCF12A cells. Reexpression of miR-200c significantly diminished ZEB1, KLF4, and BMI1 expression but restored E-cadherin expression which was repressed by knocking-down p53 (Fig. 3a). These results indicated that p53 may modulate EMT through up-regulating miR-200c. To further investigate whether p53 down-regulates stem cell properties through modulating miR-200c, we expressed precursor miR-200c in p53 knocked-down MCF12A cells. Re-expression of miR-200c significantly diminished the percentage of CD24-CD44+ cells (Fig. 3b) and formation of mammospheres (Fig. 3c), both of which were enhanced by knocking-down p53 (Fig. 3b-3c). Similarly, to further examine if inhibiting miR-200c could rescue the effects of expressing p53, we applied miR-200c-antagomiR in p53 expressed-HMEC-snail cells. We showed that miR200c-antagomiR counteracts the effects of expressing p53 as it reduced the level of the epithelial cell marker E-cadherin that was increased by p53 expression (Fig. 3d). miR200c-antagomiR also restored expression levels of ZEB1, BMI1, and KLF4 (Fig. 3e) and enhanced the $\mathrm{CD} 24^{-} \mathrm{CD} 44^{+}$population (Fig. 3f), all of which were diminished by p53 expression (Fig. 3e-3f). Taken together, these observations indicate that p53 regulates EMT and stemness through the regulation of miR-200c.

Since p53 is an essential tumor suppressor and EMT-stemness has also been implicated as an important player involving tumorigenesis, we next inquire the role of p53-miR200c regulation in human cancer cells. First we overexpressed p53 mutants that were previously identified from various breast cancer samples 6 in normal MCF12A cell line. We found that opposite from the effects of expressing wild-type p53 (Fig. 4a, left), expression of these p53 mutants leads to decreased miR-200c expression (Fig. 4b), increased ZEB1, N-cadherin, and BMI1 levels (Fig. 4a, right), and also enhanced $\mathrm{CD} 24^{-} \mathrm{CD} 44^{+}$cell population (Fig. $4 \mathrm{c}$ ). To examine whether the decrease in miR-200c by overexpressing mutated p53 is caused by impaired binding of p53 to miR-200c promoter, we performed ChIP assay using a p53 antibody that recognizes both wild-type p53 and mutated p53. Overexpression of mutated 
p53 significantly inhibited bound p53 on miR-200c promoter (Fig. 4d). Furthermore, consistent with the results of expressing p53 in normal cell lines, overexpression of wildtype p53 in breast cancer cell line BT549 (harboring p53 R249S mutant) increased miR-200c and decreased ZEB1 and BMI1 levels (Fig. 4e and 4f). However, knocking-down mutated p53 did not alter expression of either miR-200c, ZEB1 or BMI1 in BT549 cells. These data suggest that it is the wild-type p53 but not the mutated p53 that upregulates miR-200c. Together, our results suggest that mutated p53 could interfere with wild-type p53 activity as indicated by previous studies ${ }^{7}-9$ and thereby counteracts wild-type $\mathrm{p} 53$-mediated regulation on ZEB1 and BMI1 expression through modulation of miR-200c. However, overexpression or activation of wild-type p53 in cancer cell lines may override the mutated p5 $3^{10}$ and enforce the promotion of miR-200c to block EMT and stem cell properties.

To provide physiological evidence for p53 regulation on EMT and stem cell properties, we collected mammary tissues from two p53 knock-out mice and their wild-type counterparts (Fig. 5a). Consistent with our observations of knocking-down p53 in human mammary epithelial cells, mouse mammary cells knocked-out p53 exhibited elevated expression ZEB1, N-cadherin (Fig. 5b) and BMI1 (Fig. 5c) along with significant reduction of miR-200c level (Fig. 5c) compared to the wild-type counterparts. To further strengthen the pathological correlation between p53 and EMT-stem cell-associated markers in breast cancer, we performed a correlation analysis of p53, ZEB1, E-cadherin, and BMI1 protein expression levels and miR-200c miRNA level using immunohistochemistry and in situ hybridization respectively in breast cancer tissue microarrays consisting of 106 breast tumor samples (Fig. 5d and 5e). Consistently, loss of p53 correlated positively with increased ZEB1 and BMI1 expression levels but negatively with miR-200c and E-cadherin levels (Fig. $5 \mathrm{~d}$ and $5 \mathrm{e}$ ). Loss of p53 also correlated positively with poor differentiation in high grade tumors (Fig. 5f, upper) in which miR-200c expression level was significantly reduced compared to the well-differentiated tumors (Fig. 5f, lower). Together, these data suggest loss of p53 could lead to downregulation of miR-200c and activation of EMT and stemness signature that contribute to breast cancer progression.

In this study, we first used promoter analysis and miRNA array profiling to identify a link between p53 and miRNAs in the regulation of both EMT and stem cell properties.

Furthermore, physiological induction of EMT, such as TGF- $\beta$ treatment, on one hand, could inhibit 553 expression by increasing MDM $2^{11}$. On the other hand, TGF- $\beta$ activates downstream ZEB1 transcription suppressors that repress epithelial regulator E-cadherin and miR-200c to proceed to EMT program (Supplementary Information, Fig. S1a-c). Activation of p53 and its transcriptional target miR-200c led to MET and differentiation phenotype as a result of restored E-cadherin expression and repressed stemness-associated BMI1expression (Fig. 5g). In the tumor cells deficient in p53 or with constitutively active TGF- $\beta$ signaling, miR-200c targets, BMI1 and KLF4, are constitutively activated in favor to maintain stemness and generate stem cell-like population (Fig. 5g). However, this process can be reversed in normal cells as we found that upon removal of TGF- $\beta$ from MCF12A cells pretreated with TGF- $\beta$ for 14 days, p53 expression level was readily restored in 4 days, accompanied by markedly increased expression of epithelial marker E-cadherin and reduced CD $24^{-} \mathrm{CD} 44^{+}$stem cell population (Supplementary Information, Fig. S3b). 
It is known that EMT plays an essential role in cancer metastasis and restoration of MET program should efficiently slow dissemination of tumor cells ${ }^{5}$. Many molecules have been shown to mediate EMT, but the regulators that contribute to MET, especially in adult normal or neoplastic epithelial models, remain to be elucidated. From our results, p53 overexpression in HMEC-snail reverts the mesenchymal phenotype to epithelial phenotype (Supplementary Information, Fig. S4a) without inducing significant effects in cell senescence, cell death or cell cycle regulation (Supplementary Information, Fig. S4a and S4b). Our study endows p53 a role in regulating EMT-MET plasticity and potentially in cancer metastasis as a safe guard for maintaining differentiated epithelial cell phenotype independent of its conventional function.

Furthermore, stemness properties of normal stem cells are often shared with stem-like cells within tumors termed "tumor initiating cells (TICs). These TICs are shown to account for tumor initiation, progression, and chemo-resistance ${ }^{12}$. Thus, our results suggest that the $\mathrm{p} 53-$ miR-200c pathway most likely accounts for regulating EMT-associated stem cell or tumor stem cell population and activation of the p53-miR-200c regulatory axis may serve as a therapeutic alternative for targeting EMT associated-TICs.

\section{Methods}

\section{Cell culture and treatment}

Immortal normal mammary epithelial cells, MCF12A, were grown in DMEM-F12 medium supplemented with $5 \%$ horse serum, penicillin $(50 \mathrm{U} / \mathrm{ml})$, streptomycin $(50 \mathrm{U} / \mathrm{ml})$, epidermal growth factor (EGF, $20 \mathrm{ng} / \mathrm{ml})$, insulin $(10 \mu \mathrm{g} / \mathrm{ml})$, cholera toxin $(1 \mathrm{ng} / \mathrm{ml})$ and hydrocortisone. Primary human mammary epithelial cells (HMECs), HMEC-Snail cells that overexpress Snail are kind gifts from Dr. Robert A. Weinberg, were cultured in MEGMR Mammary Epithelial Cell Growth Medium (Lonza Inc.). For TGF- $\beta$ treatment, MCF12A cells were treated with $20 \mathrm{ng} / \mathrm{ml}$ of TGF- $\beta 1$ (Invitrogen) for the indicated days. miR-200c precursor and miR-200c antagomir $(50 \mathrm{nM})$ targeting endogenous miR-200c were purchased from Ambion.

\section{Plasmid construction}

Retroviral contructs pMKO.1-puro-p53 shRNAs, lentiviral constructs which direct the expression of wide type p53 (pLenti6-V5-p53_wt p53), p53 mutants (pLenti6-V5p53R175H, pLenti6/V5-p53R249S, pLenti6-V5-p53R273H, and pLenti6-V5-p53R280K) ${ }^{6}$ and shRNA against p53 (pLKO-p53-shRNA-427 and pLKO-p53-shRNA-941) ${ }^{13}$ and their control vectors were purchased from Addgene. To obtain miR-183-Luc or miR-200C-Luc, a PCR-derived fragment from miR-200c or miR-183 promoter was inserted into XhoI- and HindIII-treated pGL3 vector (Promega). To generate mutated miR-183-Luc or miR-200cLuc construct that harbors mutations in the putative p53 response elements, a PCR-based site-directed mutagenesis (Stratagene) was used. The retroviral expression vector pBMNp53 was constructed by inserting an EcoRI-SalI treated fragment of the p53 gene derived by RT-PCR into EcoRI-XhoI treated pBMN-I-GFP vector (purchased from Addgene). All of these constructs were verified by DNA sequencing. 


\section{Promoter analysis}

Analysis of putative transcription factor binding sites on miR-183 and miR-200c promoter was done by TRED (Cold Spring Harbor Laboratory) and Genomatix software.

\section{MicroRNA array}

miRNAs extracted and purified from CD24-CD44 ${ }^{+}$(stem) and non-CD24 ${ }^{-} \mathrm{CD} 44^{+}$(nonstem) cell populations isolated from primary human mammary epithelial cells (HMECs) and normal mammary epithelial cell line (MCF12A) using flow cytometry. Total RNA was prepared using RNeasy Plus Mini kit (Qiagen) following the manufacturer's protocol. Target labeling, hybridization, imaging and data processing were performed by genomic facility at MD Anderson Cancer Center using miRCURY LNA ${ }^{\mathrm{TM}}$ microRNA Array, 5th generation platform (Exiqon).

\section{Generation of stable p53-expressed and knocked-down cell lines}

For reteroviral infection, Phoenix ${ }^{\text {TM }}$-Ampho cells (Allele Biotechnology) were used for virus package according to the user instruction. Briefly, retroviral constructs such as pMKO. 1-puro-p53shRNAs or pBMN-p53 was transfected into Phoenix ${ }^{\mathrm{TM}}$-Ampho cells, and viral particles were harvested at 24 and $48 \mathrm{hr}$ post-transfection. HMEC or MCF12A cells were infected with virus for $24 \mathrm{hrs}$ twice in the presence of polybrene $(5 \mu \mathrm{g} / \mathrm{ml})$. To obtain p53shRNA knock-down cell line (shp53) or control cell line (shVec), MCF12A cells infected with pMKO.1-puro-p53shRNA (shp53: GACTCCAGTGGTAATCTACTG) or control vectors were subsequently selected with puromycin $(2 \mu \mathrm{g} / \mathrm{ml})$ for 2 weeks, and the expression of p53 was examined by Western blotting. For lentiviral infection, the third generation lentivirus packaging system was used ${ }^{14}$. Briefly, lentiviral construct that expresses wild-type p53, p53 mutants or shRNAs targeting p53

(shp53-1:CACCATCCACTACAACTACAT, shp53-2:GTCCAGATGAAGCTCCCAGAA) was co-transfected with lentiviral packaging plasmids: pMDLg-pRRE, PRSV-Rev, and PMD2G into 293T cells by Lipofectamine ${ }^{\mathrm{TM}} 2000$ according to the manufacturer's instruction. At 24 and $48 \mathrm{hr}$ post-transfection, culture medium was collected to be incubated with target cells in the presence of polybrene (see above). At $48 \mathrm{hr}$ post-infection, infected cells were harvested for gene and protein expression analysis or selected with Puromycin $(2 \mu \mathrm{g} / \mathrm{ml})$ or Blasticidin $(10 \mu \mathrm{g} / \mathrm{ml})$ for 2 weeks to establish stable clones.

\section{Flow cytometry analysis}

The FITC-conjugated anti-CD44 (BD Biosciences), APC-conjugated anti-CD44 (BD Biosciences), PE-conjugated anti-CD24 (BD Biosciences) or PerCP-Cyt5.5 anti-Human CD324 (E-Cadherin, Biolegend) antibodies were used for FACS analysis. PI staining for cell cycle analysis was performed according to the standard protocol. To obtain $\mathrm{CD} 44^{+} \mathrm{CD} 24^{-}$cells, HMEC or MCF12A cells stained with FITC-conjugated anti-CD44 and PE-conjugated anti-CD24 antibody were subjected to cell sorting performed by the Flow Cytometry and Cellular Imaging Core Facility at MD Anderson Cancer Center. 
ChIP assay

Chromatin immunoprecipitation experiment was modified from the EZ-CHIP (Upstate) protocol using p53 antibody (Santa Cruz). Primer sequences used were described in Burk et al. ${ }^{15}$.

\section{Luciferase reporter assay}

Cells were transfected with reporter plasmid, miR-183-Luc or miR-200c-Luc, and the TKRenilla reporter (Promega) was also cotransfected for normalization of transfection efficiency. Transfected cells were treated with etoposide (Sigma) and harvested at $48 \mathrm{hr}$ post-transfection, and a dual luciferase assay was performed according to the manufacturer's instruction (Promega). The luciferase activity was measured with an AutoLumat LB953 luminometer (Berthold).

\section{Real-time PCR}

Total RNAs were extracted from cells by using RNeasy kit (Qiagen). RNAs were reversetranscribed by using Superscript II kit (Invitrogen). The results were analyzed by the iCycler (Biorad), and the quantification of RNA levels was normalized to GAPDH as CT (difference of cycling threshold $)=\mathrm{CT}$ (target)-CT (control). Higher $\mathrm{CT}$ values indicate relatively lower expression RNA levels. Primer sequences were used as follows: p53Exon2-

CATCACCTCACTGCATGGAC (forward), p53Exon2- AAGCCATAGTTGCCCTGGTA (reverse), and also were described in Wellner et al. ${ }^{4}$.

\section{Senescence assay}

Cell senescence was measured by $\beta$-Galactosidase Staining kit (Cell Signaling) following the manufacturer's protocol.

\section{Antibodies}

The following antibodies were purchased commercially: anti-ZEB1 (D80D3, Cell signaling Tech.), anti-p53 (DO-1, Santa Cruz), anti-E-Cadherin (Santa Cruz), anti-N-cadherin (Ab76057, Abcam), anti-Vimentin (Santa Cruz), anti-BMI1 (Bethyl Laboratories), FITCconjugated anti-CD44 (BD Biosciences), APC-conjugated anti-CD44 (BD Biosciences), PEconjugated anti-CD24 (BD Biosciences), and PerCP-Cy5.5 anti-Human CD324 (ECadherin, Biolegend).

\section{p53 knockout mice}

Mammary tissues were dissected from p53 knockout mice and the wild-type counterparts (gifts from Dr. Elsa Flores). Information on the p53 knockout mice was described in Jacks et al. ${ }^{16}$. p53 deletion was validated by detecting p53 Exon2 mRNA expression using RT-PCR.

\section{Tissue microarray}

Breast cancer tissue microarrays (BRC1021, Figure 5e; BRC1502 and BRC1503, Figure 5f) were purchased from Pantomics. Each array includes more than 100 cases of normal, reactive, premalignant and malignant tissues of the breast (various grades and stages). 


\section{Immunocytochemistry and in situ hybridization}

Immunohistochemical staining was performed as previously described ${ }^{17}$ using anti-p53 anitbody that preferentially recognizes wild-type p53 (PAb1620, Calbiochem) ${ }^{18}{ }^{20}$, antiBMI1 (R\&D Systems), ZEB1 (Bethyl Laboratories), and E-cadherin (Cell Signaling). In situ hybridization was performed using miRNA-200c probe from Exiqon (miRCURY LNA detection probe $5^{\prime}$-DIG labeled).Detection of the probe was carried out bydigoxigenin antibody (21H8) (1:200, ab420, Abcam), LSAB2 System-HRP (K0672, Dako) and liquid DAB+ Substrate Chromogen System (K3468, Dako) following manufacturer's protocols. The correlation between 533 and ZEB1, BMI1, E-cadherin, miR-200c was analyzed using Chi-Square test.

\section{Statistical analysis}

All data were presented as means \pm the standard deviation of the mean (SD). Statistical calculations were performed with Microsoft Excel analysis tools. Differences between individual groups were analyzed by paired $t$ test. $P$ values of $<0.05$ considered statistically significant.

\section{Supplementary Material}

Refer to Web version on PubMed Central for supplementary material.

\section{Acknowledgments}

We thank Dr. Robert Weinberg (Whitehead institute, MIT) and Dr. SenduraiMani (UT MD Anderson Cancer Center) for providing HMEC and HMEC-Snail cells, Dr. Elsa Flores for providing the p53 knockout mice, and Dr. Jingyu Lang for technical assistance. This study was supported by RO1 CA109311, PO1 CA099031, Breast SPORE P50 CA116199, Cancer Center Support Grant CA16672, National Breast Cancer Foundation, Inc., Breast Cancer Research Foundation, Sister Institution Fund of China Medical University Hospital and MD Anderson Cancer Center, National Science Council of Taiwan NSC-96-3111-B-039, NSC-2632-B-039 and Cancer Research Center for Excellence, Taiwan Department of Health, DOH99-TD-C-111-005 (to M-C. H.). In memoriam, Ms. Serena Lin-Guo for her courageous fight against cancer.

\section{References}

1. Mani SA, et al. The epithelial-mesenchymal transition generates cells with properties of stem cells. Cell. 2008; 133:704-715. [PubMed: 18485877]

2. Polyak K, Weinberg RA. Transitions between epithelial and mesenchymal states: acquisition of malignant and stem cell traits. Nat Rev Cancer. 2009; 9:265-273. [PubMed: 19262571]

3. Shimono Y, et al. Downregulation of miRNA-200c links breast cancer stem cells with normal stem cells. Cell. 2009; 138:592-603. [PubMed: 19665978]

4. Wellner U, et al. The EMT-activator ZEB1 promotes tumorigenicity by repressing stemnessinhibiting microRNAs. Nat Cell Biol. 2009; 11:1487-1495. [PubMed: 19935649]

5. Thiery JP, Sleeman JP. Complex networks orchestrate epithelial-mesenchymal transitions. Nat Rev Mol Cell Biol. 2006; 7:131-142. [PubMed: 16493418]

6. Junk DJ, et al. Different mutant/wild-type p53 combinations cause a spectrum of increased invasive potential in nonmalignant immortalized human mammary epithelial cells. Neoplasia. 2008; 10:450 461. [PubMed: 18472962]

7. Willis A, Jung EJ, Wakefield T, Chen X. Mutant p53 exerts a dominant negative effect by preventing wild-type p53 from binding to the promoter of its target genes. Oncogene. 2004; 23:2330-2338. [PubMed: 14743206] 
8. Cadwell C, Zambetti GP. The effects of wild-type p53 tumor suppressor activity and mutant p53 gain-of-function on cell growth. Gene. 2001; 277:15-30. [PubMed: 11602342]

9. Milner J, Medcalf EA, Cook AC. Tumor suppressor p53: analysis of wild-type and mutant p53 complexes. Mol Cell Biol. 1991; 11:12-19. [PubMed: 1986215]

10. Nielsen LL, et al. Efficacy of p53 adenovirus-mediated gene therapy against human breast cancer xenografts. Cancer Gene Ther. 1997; 4:129-138. [PubMed: 9080122]

11. Araki S, et al. TGF-beta1-induced expression of human Mdm2 correlates with late-stage metastatic breast cancer. J Clin Invest. 120:290-302. [PubMed: 19955655]

12. Al-Hajj M, Clarke MF. Self-renewal and solid tumor stem cells. Oncogene. 2004; 23:7274-7282. [PubMed: 15378087]

13. Kim JS, Lee C, Bonifant CL, Ressom H, Waldman T. Activation of p53-dependent growth suppression in human cells by mutations in PTEN or PIK3CA. Mol Cell Biol. 2007; 27:662-677. [PubMed: 17060456]

14. Dull T, et al. A third-generation lentivirus vector with a conditional packaging system. J Virol. 1998; 72:8463-8471. [PubMed: 9765382]

15. Burk U, et al. A reciprocal repression between ZEB1 and members of the miR-200 family promotes EMT and invasion in cancer cells. EMBO Rep. 2008; 9:582-589. [PubMed: 18483486]

16. Jacks T, et al. Tumor spectrum analysis in p53-mutant mice. Curr Biol. 1994; 4:1-7. [PubMed: 7922305]

17. Xia W, et al. Phosphorylation/cytoplasmic localization of $\mathrm{p} 21 \mathrm{Cip} 1 / \mathrm{WAF} 1$ is associated with HER2/neu overexpression and provides a novel combination predictor for poor prognosis in breast cancer patients. Clin Cancer Res. 2004; 10:3815-3824. [PubMed: 15173090]

18. Kramata $P$, et al. Patches of mutant p53-immunoreactive epidermal cells induced by chronic UVB Irradiation harbor the same p53 mutations as squamous cell carcinomas in the skin of hairless SKH-1 mice. Cancer Res. 2005; 65:3577-3585. [PubMed: 15867351]

19. Muret J, et al. p53 status correlates with histopathological response in patients with soft tissue sarcomas treated using isolated limb perfusion with TNF-alpha and melphalan. Ann Oncol. 2008; 19:793-800. [PubMed: 18065405]

20. Xirodimas DP, Lane DP. Molecular evolution of the thermosensitive PAb1620 epitope of human p53 by DNA shuffling. J Biol Chem. 1999; 274:28042-28049. [PubMed: 10488156] 
a

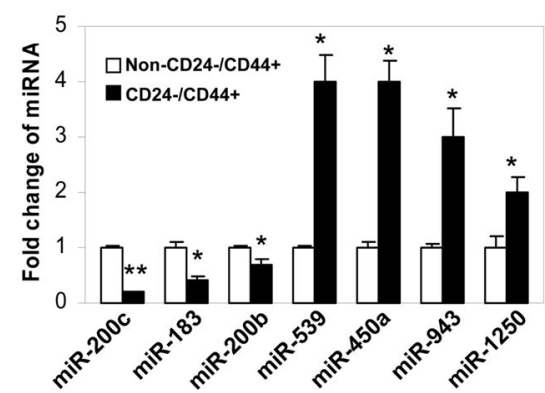

b

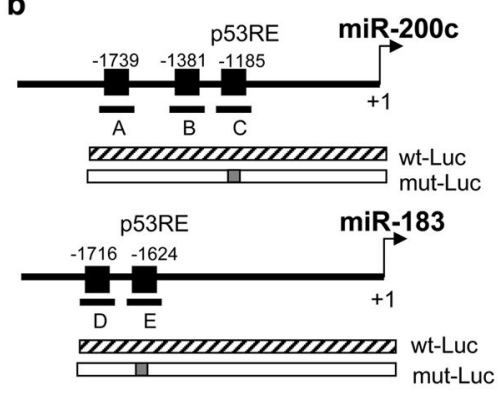

C

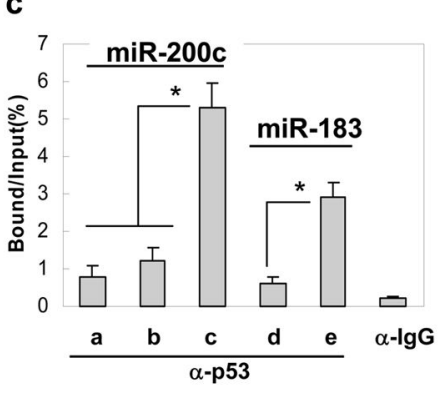

d
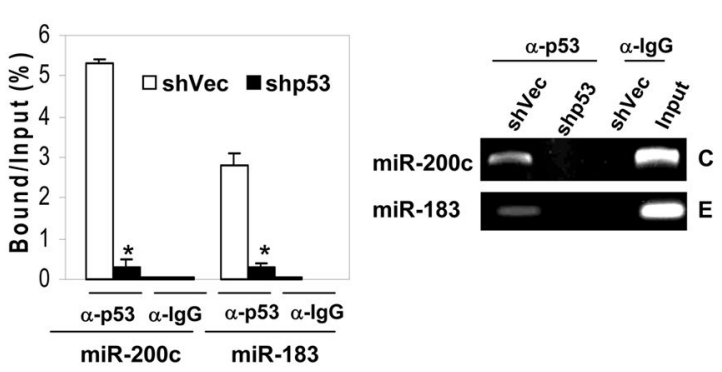

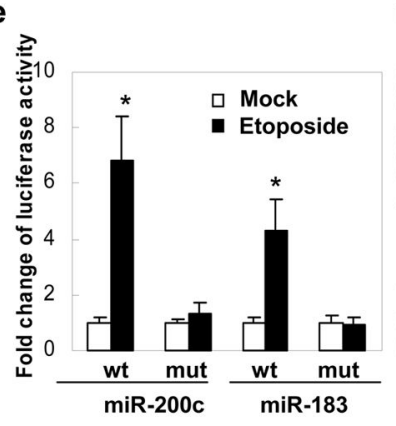

f

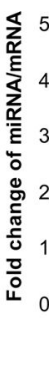

g

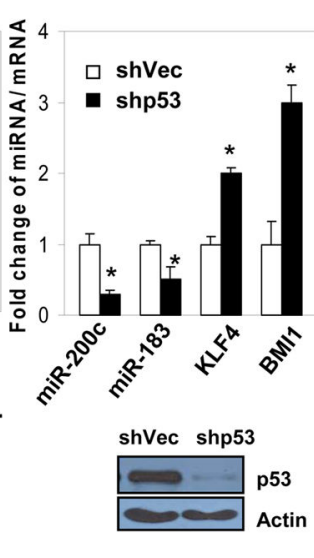

Figure 1. p53 transcriptionally activates miR-183 and miR-200c

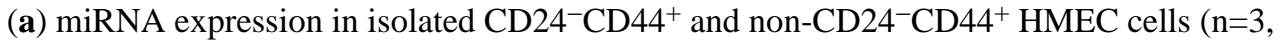
double asterisk indicates $\mathrm{P}<0.01$, single asterisk indicates $\mathrm{P}<0.05$ ). (b) Diagram showing the promoter regions of miR-183 and miR-200c with the putative p53 response elements (p53$\mathrm{RE}$ ), and the structure of wild-type and p53RE-mutant luciferase reporters driven by the indicated promoters. (c) The percentage of the bound chromatin/input chromatin using p53 antibody targeting p53 binding elements (A-E) in HMEC cells. IgG was used as a negative control. ( $\mathrm{n}=3$, asterisk indicates $\mathrm{P}<0.05$ ). (d) ChIP-PCR gel (left) and the percentage (right) of the bound chromatin/input chromatin using p53 antibody in MCF12A cells ( $\mathrm{n}=3$, asterisk indicates $\mathrm{P}<0.05$ ). (e) The fold change of luciferase activity driven by wild-type and mutant p53 response elements under etoposide treatment $(\mathrm{n}=5$, asterisk indicates $\mathrm{P}<0.05)$. $(\mathbf{f}-\mathbf{g})$ miRNA or mRNA expression levels in cells expressing the indicated constructs $(n=4$, 
asterisk indicates $\mathrm{P}<0.05)$. Insets showing p53 protein expression levels. Error bars denote \pm SD. 
a

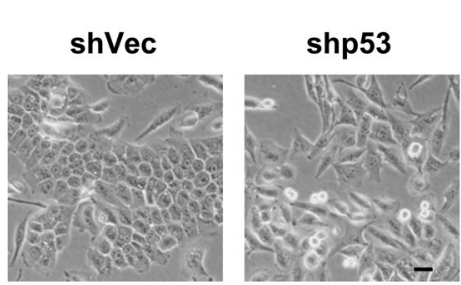

C

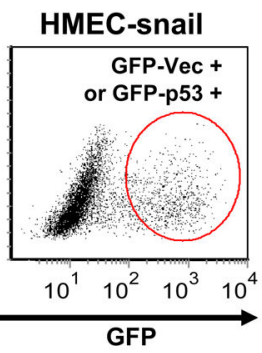

b
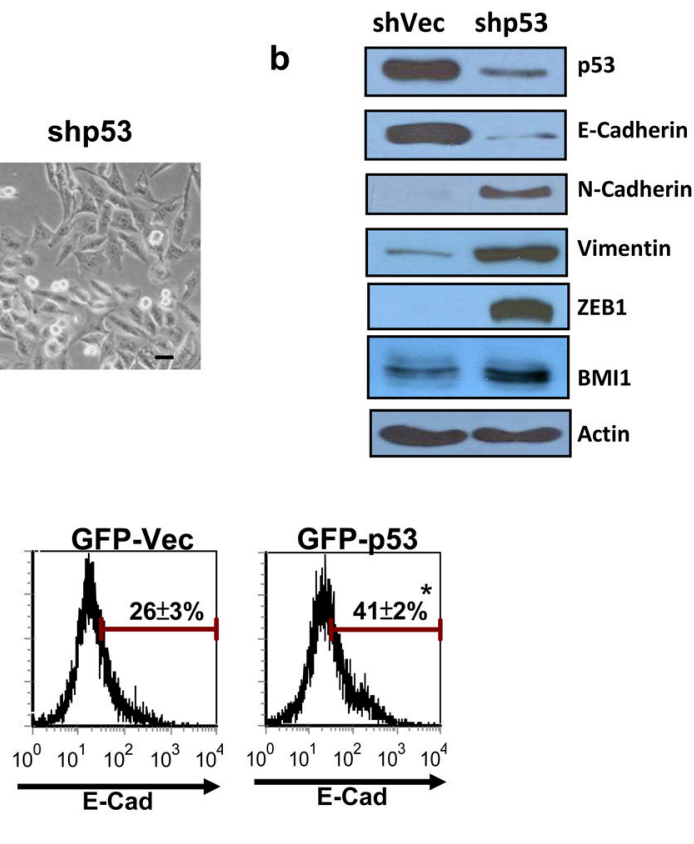

d

shVec

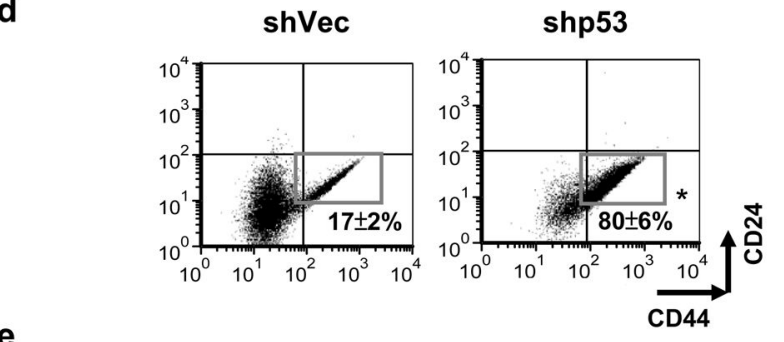

e
HMEC-snail

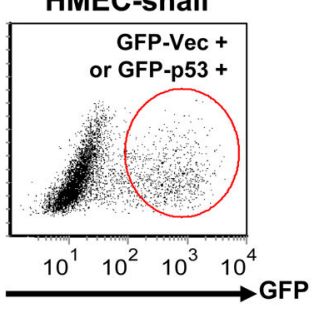

GFP-Vec

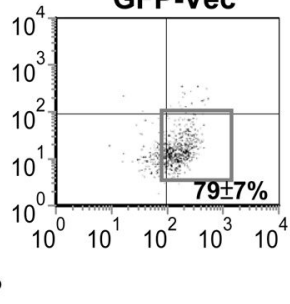

GFP-p53

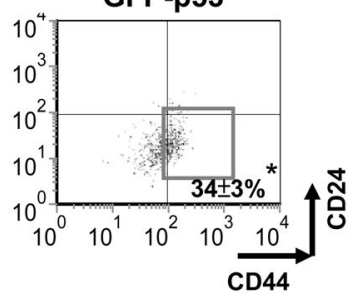

Figure 2. p53 suppresses EMT phenotype and stem cell properties

(a) Images of the cell morphology of MCF12A p53 knocked-down (scale bar: $20 \mu \mathrm{m}$ ). (b) Protein expression levels of MCF12A p53 knocked-down cells. (c) Flow plots showing Ecadherin expression in sorted HMEC-Snail cells expressing GFP-p53 (circle) ( $\mathrm{n}=3$, asterisk indicates $\mathrm{P}<0.05$ ). (d) Flow plots showing percentage of $\mathrm{CD} 24^{-} \mathrm{CD} 44^{+}$population (lower right box) in MCF12A p53 knocked-down cells ( $\mathrm{n}=3$, asterisk indicates $\mathrm{P}<0.05)$. (e) Flow charts showing the percentage of $\mathrm{CD} 24^{-} \mathrm{CD} 44^{+}$population (lower right box) in sorted HMEC-Snail cells expressing GFP-p53 (circle) $(\mathrm{n}=3$, asterisk indicates $\mathrm{P}<0.05)$. Error bars denote \pm SD. 
a

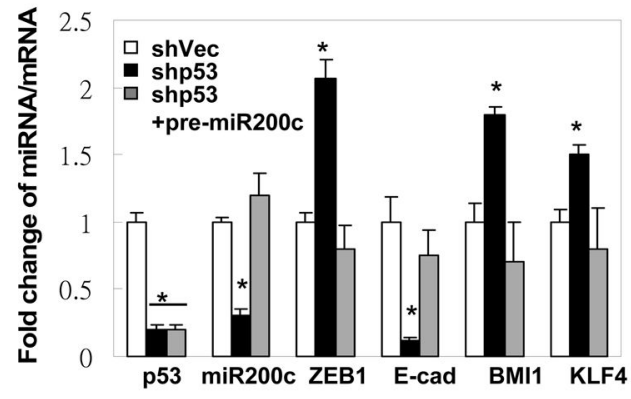

b

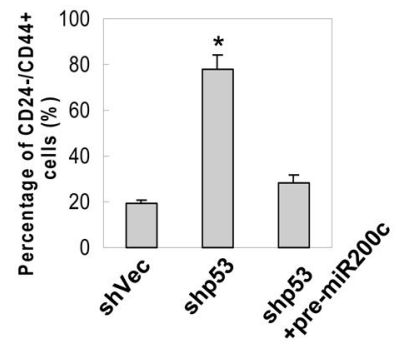

C

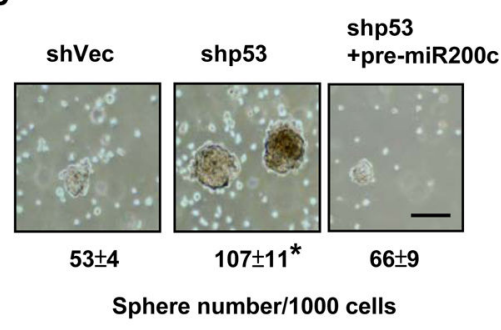

d
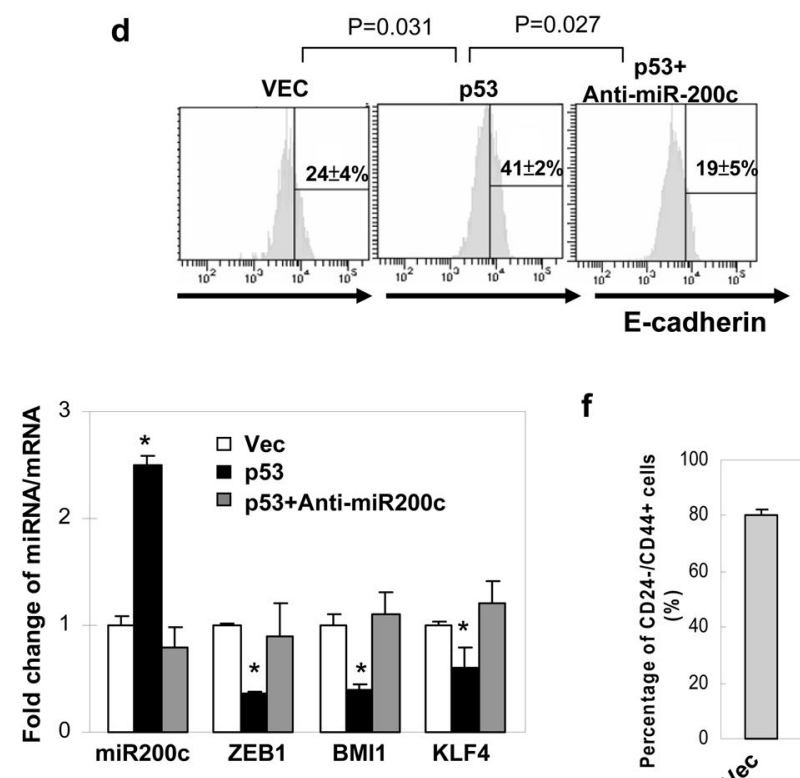

f

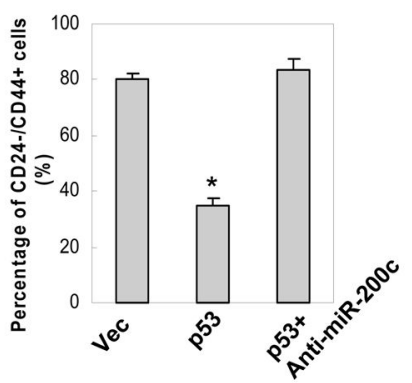

Figure 3. p53 suppresses EMT phenotype and stem cell properties through upregulating miR-200c

(a) miRNA or mRNA expression levels in MCF12A cells expressing the indicated constructs $(n=4$, asterisk indicates $\mathrm{P}<0.05)$. (b) The percentage of $\mathrm{CD} 24^{-} \mathrm{CD} 44^{+}$population in MCF12A cells expressing the indicated constructs $(\mathrm{n}=3$, asterisk indicates $\mathrm{P}<0.05)$. (c) Images showing mammosphere formation in MCF12A cells expressing the indicated constructs. Number of spheres were counted for 10 fields in each group (scale bar: $100 \mu \mathrm{m}$, $\mathrm{n}=3$, asterisk indicates $\mathrm{P}<0.05$ ). (d) Flow plots showing E-cadherin expression in HMECSnail cells expressing the indicated constructs $(\mathrm{n}=3$, asterisk indicates $\mathrm{P}<0.05)$. (e) miRNA or mRNA expression levels in HMEC-Snail cells expressing the indicated constructs $(n=4$, asterisk indicates $\mathrm{P}<0.05$ ). (f) The percentage of $\mathrm{CD} 24^{-} \mathrm{CD} 44^{+}$population in HMEC-Snail 
cells expressing the indicated constructs $(\mathrm{n}=3$, asterisk indicates $\mathrm{P}<0.05)$. Error bars denote \pm SD. 

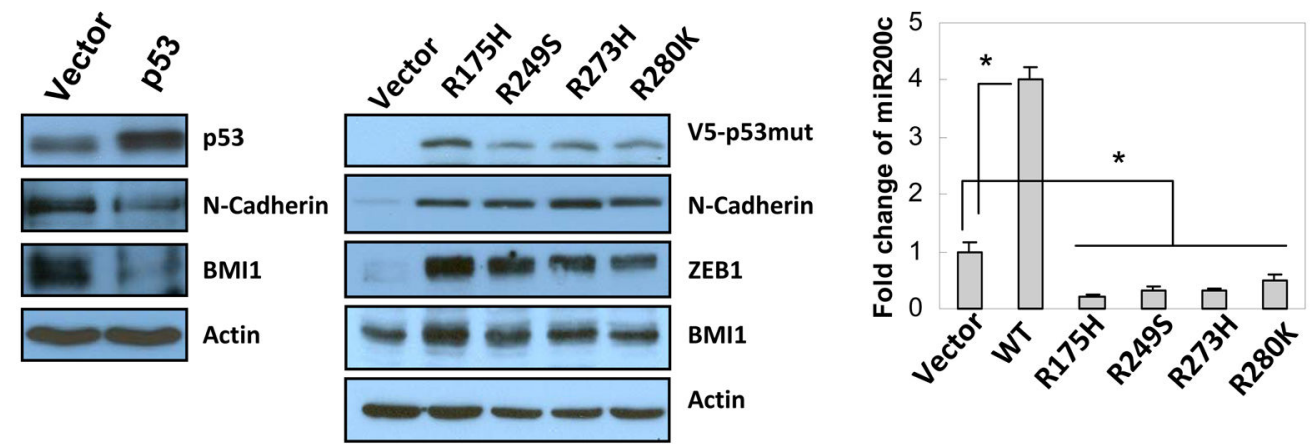

C

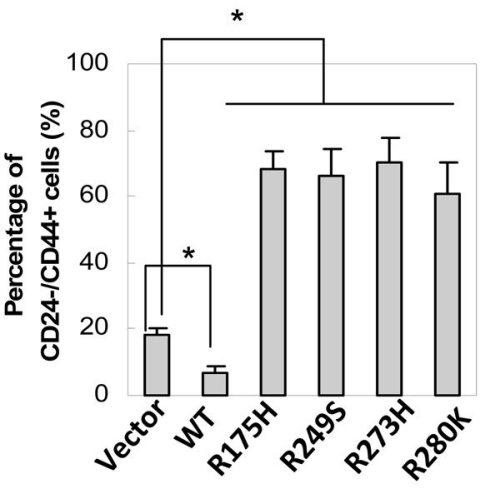

e $\quad$ BT -549

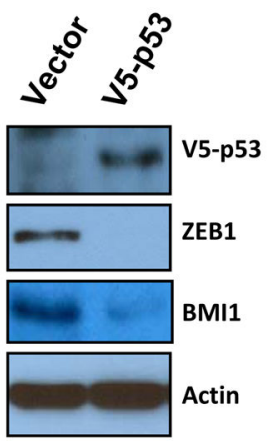

d

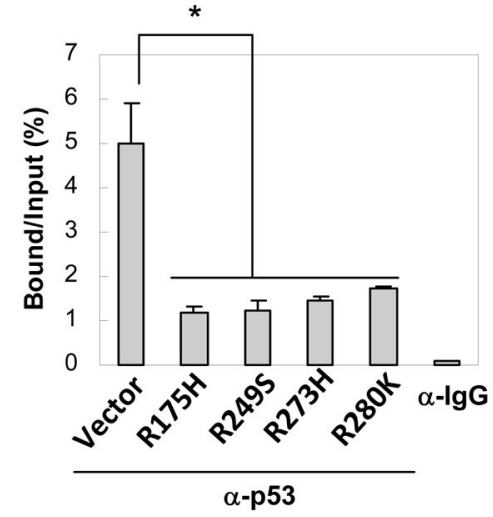

$\mathbf{f}$

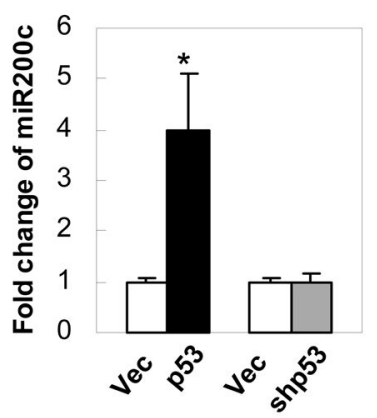

Figure 4. Mutated p53 competes with wild-type p53 in the regulation of EMT and stemness markers

(a) Protein expression levels of MCF12A cells expressing the indicated constructs. (b) miRNA-200c expression levels of MCF12A cells expressing the indicated constructs ( $\mathrm{n}=4$, asterisk indicates $\mathrm{P}<0.05$ ). (c) The percentage of $\mathrm{CD} 24^{-} \mathrm{CD} 44^{+}$population in MCF12A cells expressing the indicated constructs $(\mathrm{n}=3$, asterisk indicates $\mathrm{P}<0.05)$. (d) The percentage of the bound chromatin over input chromatin immunoprecipitated by 553 antibody in MCF12A cells expressing the indicated constructs. IgG was used as a negative control $(\mathrm{n}=3$, asterisk indicates $\mathrm{P}<0.05)$. (e) Protein expression levels of BT-549 breast cancer cells expressing the 
indicated constructs. (f) Fold change in miRNA-200c expression level in BT-549 cells expressing the indicated constructs $(\mathrm{n}=4$, asterisk indicates $\mathrm{P}<0.05)$. Error bars denote \pm SD. 

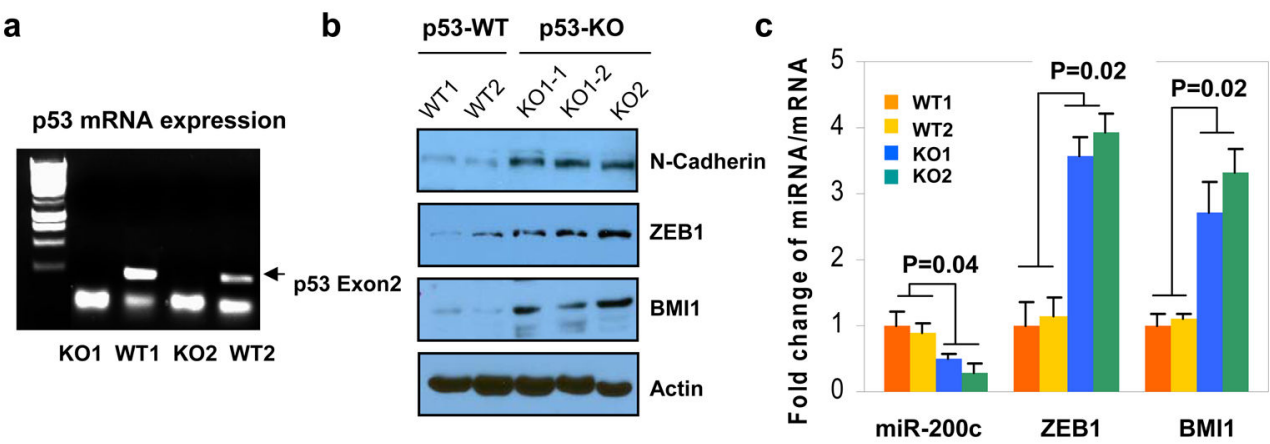

d

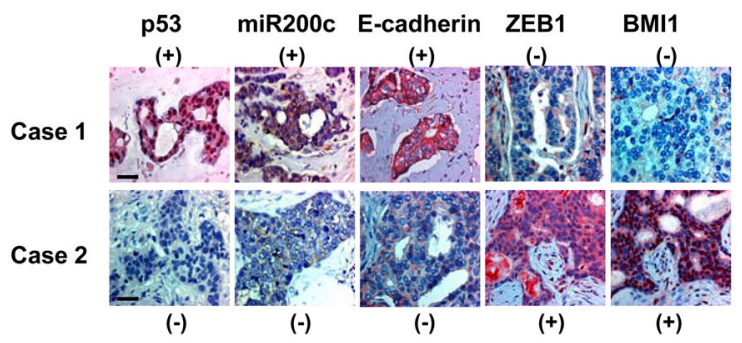

e

\begin{tabular}{|c|c|c|c|c|c|c|c|c|}
\hline \multirow[b]{2}{*}{ p53 } & \multicolumn{2}{|c|}{$\mathrm{miR} 200 \mathrm{c}$} & \multicolumn{2}{|c|}{ ZEB1 } & \multicolumn{2}{|c|}{ E-cadherin } & \multicolumn{2}{|c|}{ BMI1 } \\
\hline & - & + & - & + & - & + & - & + \\
\hline - & $\begin{array}{c}57 \\
(54 \%)\end{array}$ & $\begin{array}{c}6 \\
(5 \%)\end{array}$ & $\begin{array}{c}19 \\
(18 \%)\end{array}$ & $\begin{array}{c}44 \\
(42 \%)\end{array}$ & $\begin{array}{c}46 \\
(44 \%)\end{array}$ & $\begin{array}{c}17 \\
(16 \%)\end{array}$ & $\begin{array}{c}28 \\
(26 \%)\end{array}$ & $\begin{array}{c}35 \\
(33 \%)\end{array}$ \\
\hline+ & $\begin{array}{c}15 \\
(14 \%)\end{array}$ & $\begin{array}{c}28 \\
(27 \%)\end{array}$ & $\begin{array}{c}27 \\
(25 \%)\end{array}$ & $\begin{array}{c}16 \\
(15 \%)\end{array}$ & $\begin{array}{c}12 \\
(11 \%)\end{array}$ & $\begin{array}{c}31 \\
(29 \%)\end{array}$ & $\begin{array}{c}33 \\
(31 \%)\end{array}$ & $\begin{array}{c}10 \\
(9 \%)\end{array}$ \\
\hline Total & $\begin{array}{c}72 \\
(68 \%)\end{array}$ & $\begin{array}{c}34 \\
(32 \%)\end{array}$ & $\begin{array}{c}46 \\
(43 \%)\end{array}$ & $\begin{array}{c}60 \\
(57 \%)\end{array}$ & $\begin{array}{c}58 \\
(55 \%)\end{array}$ & $\begin{array}{c}48 \\
(45 \%)\end{array}$ & $\begin{array}{c}61 \\
(57 \%)\end{array}$ & $\begin{array}{c}45 \\
(43 \%)\end{array}$ \\
\hline
\end{tabular}

f

\begin{tabular}{|c|c|c|c|}
\hline \multicolumn{2}{|c}{ Tumor grade } & \multicolumn{1}{c|}{ III } & \multicolumn{1}{c|}{ Total } \\
\hline p53+ & $17(18 \%)$ & $7(6 \%)$ & $24(24 \%)$ \\
p53- & $11(10 \%)$ & $71(66 \%)$ & $82(76 \%)$ \\
Total & $28(28 \%)$ & $78(72 \%)$ & $106(100 \%)$ \\
\hline miR200c+ & $23(22 \%)$ & $11(11 \%)$ & $34(33 \%)$ \\
miR200c- & $9(8 \%)$ & $63(59 \%)$ & $72(67 \%)$ \\
Total & $32(30 \%)$ & $74(70 \%)$ & $106(100 \%)$ \\
\hline
\end{tabular}

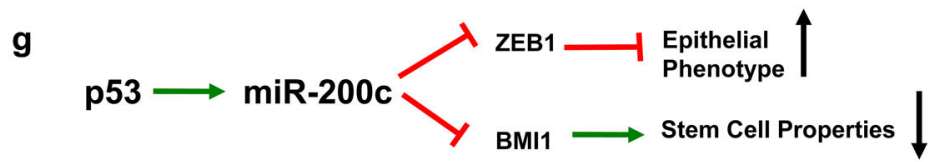

Figure 5. Loss of p53 correlates with reduced miR-200c and enhanced ZEB1 and BMI1 expression levels

(a) RT-PCR products validate the deletion of p53 (exon2) in p53 knock-out mice. (b) Protein expression levels p53 wild-type (WT1 and WT2) and p53 knock-out mice (KO1 and $\mathrm{KO} 2$ ) mammary cells. KO1-1 and KO1-2 represent samples of two mammary glands collected from the same mouse. (c) Fold change in miRNA or mRNA expression level of mammary cells from the $\mathrm{p} 53$ wild-type and 553 knock-out mice $(n=3$, asterisk indicates $\mathrm{p}<0.05$ ). Error bars denote $\pm \mathrm{SD}$. (d) Representative cases from 106 breast cancer specimens in tissue microarrays were analyzed by immunohistochemical staining (p53, E-cadherin, ZEB1, BMI1) and in situ hybridization (miR-200c) (scale bar: $20 \mu \mathrm{m}$ ). (e) (f) Tables 
summarizing Chi-Square analysis of immunohistochemical staining results. (-): negative, (+): positive. (g) A proposed model of p53 regulation on epithelial phenotype and stemness through activating miR-200c. 